\title{
Avaliação da Imagem Corporal: Notas Essenciais para uma Boa Prática de Pesquisa
}

\author{
Angela Nogueira Neves ${ }^{1}$ \\ Escola de Educação Física do Exército, Brasil \\ Fabiane Frota da Rocha Morgado \\ Maria da Consolação Gomes Cunha Fernandes Tavares \\ Universidade Estadual de Campinas
}

\begin{abstract}
RESUMO - A pesquisa em imagem corporal no Brasil teve um aumento significativo, marcadamente na última década. Apesar do inegável amadurecimento nas pesquisas atuais, ainda se identificam inconsistências metodológicas na pesquisa em Imagem Corporal, à semelhança do que ocorria no cenário exterior na década de 1990. Este artigo metodológico foca-se na análise de pontos metodológicos importantes que necessitam de atenção na proposição de uma pesquisa. As informações aqui sistematizadas foram recolhidas de artigos científicos e livros focados na pesquisa em Imagem Corporal, assim como de cursos para capacitação de pareceristas em revista especializadas nessa temática. Foram escolhidos quatro tópicos para serem abordados e cada um deles trará apontamentos relevantes para a elaboração, condução e análise dos dados da pesquisa em Imagem Corporal.
\end{abstract}

Palavras-chaves: imagem corporal, pesquisa, metodologia

\section{Body Image Assessment: Essential Notes for a Good Research Practice}

\begin{abstract}
Body image research had a significant growth in Brazil, markedly in the last decade. Despite the undeniable maturity that the recent production is showing, some methodological inconsistencies can still been identified in body image research in Brazil, similar to what happened outside Brazil in the decade of the 1990s. This methodological article is focused on important methodological points that require attention in the phase of research planning. The information systematized here was collected from books and scientific articles, as well from courses for reviewers of journals specialized in this area. Four topics were chosen to be discussed here and each of them will bring relevant notes for elaboration, execution and data analyzes in body image research.
\end{abstract}

Keywords: body image, research, methodolog

A pesquisa em Imagem Corporal teve um importante crescimento nas últimas duas décadas, especialmente no desenvolvimento de formas de avaliação e de perspectivas conceituais. Especialmente no Brasil, nota-se um aumento no número de pesquisas, marcadamente na última década, que resultou na publicação de livros de referência para pesquisadores nacionais, uma crescente produção e artigos veiculados em revistas nacionais, aumento na projeção da produção científica Brasileira no cenário internacional e a realização de eventos voltados especificamente para a discussão desse tema. Todavia, não se deve perder de vista que a pesquisa em Imagem Corporal não é uma tarefa simples e uma análise na produção nacional evidencia alguns pontos frágeis, à semelhança do que ocorria na produção internacional, notadamente norte-americana, na década de 1990.

É importante observar que a pesquisa exige conhecimento teórico consistente, planejamento cuidadoso na definição dos instrumentos de pesquisa, rigor na coleta de dados, interpretação de dados que não considere apenas provas matemáticas (no caso de pesquisas quantitativas), mas uma

1 Escola de educação Física do Exército, Divisão de Pesquisa e Extensão Avenida João Luis Alves, s/n. Urca, Rio de Janeiro, RJ, Brasil. CEP: 22.291-090.E-mail: angelanneves@yahoo.com.br compreensão do constructo a partir das evidências geradas pelos dados, assim como uma capacidade de articular o que é relatado pelo sujeito com as evidências já estabelecidas neste campo de pesquisa, sem gerar distorções (Thompson, 2004).

Este artigo metodológico foca-se na análise de alguns pontos que necessitam de atenção na proposição de uma pesquisa em Imagem Corporal, de forma a garantir êxito na coleta de informação e coerência na discussão dos dados. As informações aqui apresentadas são oriundas de livros e artigos científicos (p.ex.: Campana \& Tavares, 2009; Cash, 2011; Fisher, 1990; Krawczyk, Menzel, \& Thompson, 2012; McCabe, Ricciardelli, Sitaram, \& Mikhail 2006; Smeets, 1997; Thompson, 1990, 2004; Thompson, Heinberg, Altabe, \& Tantleff-Dunn, 1998), assim como materiais destinados à capacitação de pareceristas de revistas especializadas nesse tema. O texto apresenta reflexões e sínteses dos autores baseadas nessas informações.

Considerando o cenário da pesquisa em Imagem Corporal no Brasil, foram escolhidos quatro tópicos para serem abordados: (1) princípios básicos; (2) escolha e adequação de instrumentos; (3) procedimentos na coleta de dados; (4) análise dos dados. Cada tópico trará apontamentos considerados importantes para a elaboração, condução e 
análise dos dados da pesquisa em Imagem Corporal, para que a mesma seja mais assertiva e menos morosa ${ }^{1}$.

\section{Princípios básicos para a pesquisa em Imagem Corporal}

É essencial à pesquisa na área de Imagem Corporal uma compreensão teórica muito bem estabelecida sobre esse tema. É também indispensável o conhecimento das diferentes perspectivas (cognitiva-comportamental, cognitiva, psicodinâmica, neurológica, sociocultural, evolucionista, feminista), as nuances de cada uma dessas perspectivas na definição teórica da Imagem Corporal, assim como suas formas específicas de investigação. O estudo, a clareza do conceito de Imagem Corporal que se adota e a atitude reflexiva constante são posturas próprias da rotina daquele que pesquisa em Imagem Corporal e podem contribuir para que a interpretação dos dados seja feita de forma consistente (Campana \& Tavares, 2009; Cash, 2011; Thompson, 2004).

Em qualquer pesquisa, estabelecer qual o referencial teórico que a suporta e o define é o primeiro passo para que os dados gerados possam ser compreendidos. Porém, na pesquisa em Imagem Corporal isso se faz especialmente importante, por haver definições operacionais diversas, ou seja, termos diversificados com a mesma definição e termos distintos com definições semelhantes (Fisher, 1990; Thompson et al., 1998). Essa clareza é imperativa para evitar que, na discussão dos dados, o pesquisador se perca e seja contraditório em relação à definição e perspectiva teórica por ele inicialmente adotada. Ademais, garante que as conclusões e afirmações, mesmo que sejam lidas por pesquisadores de outras áreas, sejam bem compreendidas.

A falta de aprofundamento teórico do conceito de Imagem Corporal e da compreensão do alcance dos vários caminhos metodológicos já disponíveis levam a afirmações equivocadas como: "é impossível avaliar Imagem Corporal" ou "há transtornos da Imagem Corporal que precisam ser consertados". Sendo a Imagem Corporal um constructo singular, dinâmico e multifacetado, é essencial saber dos limites dos dados obtidos. Não se pode perder de vista que os dados gerados através de entrevistas ou escalas, pertencem àquele contexto da avaliação. Os instrumentos de medida, em especial nas pesquisas quantitativas, revelam os traços mais marcantes, mais estáveis, daquela dimensão específica que o instrumento se propõe a avaliar. Dados quantitativos apresentam uma visualização dos traços da Imagem Corporal de uma dimensão específica de um determinado grupo, ou seja, o que aquele grupo comunga quanto à representação da identidade corporal de seus integrantes (Fisher, 1990; Tavares, 2003). Na pesquisa qualitativa, é possível compreender de forma mais particular os elementos da representação corporal do sujeito entrevistado. Ainda

1 As recomendações e observações aqui apresentadas não substituem, de forma alguma, a resolução 196/96 do Conselho Nacional de Saúde (CNS) que regulamenta as pesquisas com seres humanos no país. Os projetos de pesquisa em imagem Corporal devem ser enviados ao Comitê de Ética em Pesquisa da instituição à qual o pesquisador é credenciado - ou a que atende sua cidade, como toda pesquisa que envolva seres humanos assim, terá uma visualização parcial do constructo, pois a Imagem Corporal tem tanto elementos conscientes como inconscientes e se refere a um processo inacabado e em constante reconstrução, jamais poderá ser descoberto em definitivo $^{2}$. Em resumo, é importante sempre ter em mente que o que se revela na pesquisa é um "momento" da variável de interesse, parte da representação do corpo do sujeito. $\mathrm{O}$ pesquisador deve buscar trabalhar com a investigação concomitante de variados componentes da Imagem Corporal, a fim de obter uma visão mais detalhada da representação do corpo que se revelou naquele contexto da coleta de dados (Thompson, 2004).

\section{Escolha e Adequação dos Instrumentos}

Tendo definido qual perspectiva teórica sua pesquisa se apoia e compreendido que os dados que serão gerados na pesquisa dizem respeito ao contexto do qual serão colhidos, é importante em seguida determinar como e o que será avaliado.

Deve estar claro ao pesquisador qual dimensão (ou dimensões) da Imagem Corporal será(ão) avaliada(s). A avaliação das dimensões perceptiva e atitudinal dá-se de forma bem distinta. A avaliação da dimensão perceptiva tem por instrumentos mais ecologicamente adequados os aparatos de distorção da própria imagem do corpo do sujeito de pesquisa, seja em fotos ou em filmagens (Gardner, 2011), sustentados por fundamentos da psicofísica, que marca a reestruturação da pesquisa perceptiva na década de 1980 (Campana, 2011; Smeets, 1997; Tavares, Campana, TavaresFilho, \& Campana, 2011; Thompson, \& Gardner, 2002). $\mathrm{Na}$ avaliação da dimensão atitudinal, escalas atitudinais, escala de figuras e/ou entrevistas estruturadas são os instrumentos mais coerentes. Ainda, na dimensão atitudinal, os componentes afetivo, comportamental, cognitivo e de satisfação com o corpo tem escalas específicas para avaliação (Krawczyk et al., 2012; Menzel, Krawczyk, \& Thompson, 2011; Thompson \& Gardner, 2002). Por isso, é importante o pesquisador conhecer o leque de possibilidades de avaliação da Imagem Corporal e saber quais são aquelas mais adequadas a cada dimensão da Imagem Corporal e seus respectivos componentes. Em nosso país, esse cuidado estende-se a identificar quais estão disponíveis para serem usadas por pesquisadores brasileiros (Campana \& Tavares, 2009; Cash, 2011; Thompson, 1990, 2004).

Inserida nesta etapa de análise, está a determinação de se a pesquisa estará focada em analisar traços ou estados da Imagem Corporal. O pesquisador deve estar atento: a avaliação do imediato efeito de uma intervenção (como uma aula de ginástica, exposição às imagens midiáticas ou uma sessão de terapia) é uma avaliação de estado. Já avaliação de como os efeitos acumulativos dessas mesmas intervenções perduraram ao longo do tempo, é uma avaliação de traço. Ressalta-se que a grande maioria dos instrumentos atuais são

2 Ademais, o local e momento adequados para o próprio sujeito aprofundar-se nas questões relativas à sua identidade corporal é num processo terapêutico, num local privado na relação paciente - terapeuta, e não numa pesquisa científica. 
instrumentos para avaliação de traços de Imagem Corporal (Thompson et al., 1998).

O passo inicial para a escolha do instrumento de medida para a pesquisa é a determinação clara do que o pesquisador buscará avaliar. $\mathrm{O}$ pesquisador deve ainda se apoiar em outros aspectos, como a adequação teórica do instrumento e suas propriedades psicométricas (como validade e confiabilidade) ${ }^{3}$, um atrelado ao outro. Não se deve esquecer, nesse processo de escolha dos instrumentos para avaliação, que um instrumento de medida está localizado num referencial teórico específico, baseado em informações relevantes da literatura sobre a população alvo a que se destina ou mesmo sobre informações colhidas diretamente da população. Em resumo, o instrumento de medida, a escala, é um recorte objetivo para avaliação de uma ou mais facetas do constructo sob estudo, para um determinado grupo. Por que não perder isso de vista é relevante? Saber dos procedimentos de criação de instrumentos de avaliação impede que o pesquisador cometa erros ingênuos como, por exemplo, usar um instrumento criado para avaliar adultos em uma pesquisa na qual a amostra é composta por crianças; uma escala específica para mulheres com amostras de homens; ou ainda, uma escala criada para avaliar a Imagem Corporal de pessoas com uma condição clínica específica numa amostra de pessoas saudáveis (Campana \& Tavares, 2009; Thompson, 2004).

Inquestionável é a necessidade de a escala apresentar qualidades psicométricas satisfatórias para o contexto cultural e amostra de interesse da pesquisa. É uma exigência indiscutível nos estudos quantitativos ${ }^{4}$, em que as informações codificadas nas respostas das escalas é que serão interpretadas pela estatística inferencial. O problema de não usar um questionário adequado é que os dados podem ser questionados, pois não haverá garantias de que os resultados gerados pela pesquisa são reais ou refletem erros de medida (Cash, 2011; Krawczyk et al., 2012).

Há nessas recomendações um alerta ao pesquisador que desconhece ou que esquece o processo de criação de um instrumento, pois assume o risco de ficar trabalhando apenas num plano mais raso, rendendo-se às provas estatísticas que são incoerentes com a teoria. As evidências psicométricas de uma escala devem apresentar a confirmação observada de pressupostos teóricos. Observa-se, na produção metodológica

3 Confiabilidade refere-se à "proporção de variância atribuída ao escore verdadeiro de uma variável latente" (DeVellis, 2003, p. 27), ou de forma mais simples, a qualidade da escala de avaliar um construto livre de erros sistemáticos - seja no tempo, entre sujeito, entre pesquisadores. Validade, por sua vez, diz respeito ao "aspecto da medida ser congruente com a propriedade medida dos objetos, e não com a exatidão com que a mensuração... é feita" (Pasquali, 2003, p. 158).

4 Nos estudos qualitativos, os procedimentos são outros e devem ter também critérios mínimos de qualidade. Via de regra, o pesquisador, fundamentado por uma revisão de literatura e amparado pelo conhecimento que tem de sua população-alvo, elabora o roteiro de perguntas (questionário) que guiará sua entrevista, ou ainda, o roteiro de moderador de seu grupo focal. Submeter este questionário/roteiro ao julgamento de outros profissionais, especialistas na área, é um recurso para garantir que suas perguntas têm a capacidade de gerar informações para atingir os objetivos de sua pesquisa. Recomendamos a leitura de livros na área de pesquisa em Marketing, que descrevem esse procedimento com bastante propriedade. atual, uma rendição às evidências matemáticas, desvinculadas de um arcabouço teórico de suporte para os dados numéricos. Em estudos de validação psicométrica de escalas isso tem sido especial verdade. Assim, ao escolher a escala, o pesquisador deve analisar criticamente o(s) estudo(s) metodológico(s) da mesma (no cenário nacional e/ou internacional) a fim de verificar se determinada escala é teoricamente coerente com a teoria subjacente da Imagem Corporal (e aí voltamos ao primeiro passo de uma boa pesquisa: um conhecimento teórico consistente) e se realmente pode ser usada com a população alvo da pesquisa que se planeja realizar.

O acrônimo DIPS pode ajudar o pesquisador brasileiro a analisar aspectos na escolha do instrumento de pesquisa que comumente apresentam equívocos na produção nacional, sendo:

D: dimensão perceptiva ou atitudinal? Em sendo atitudinal, qual componente?

I: a idade de sua população é compatível com a qual o instrumento foi criado?

P: há evidências psicométricas que suportam seu uso na sua população?

S: o sexo de sua população é compatível com o qual o instrumento foi criado?

Quando o pesquisador não encontrar uma escala adequada para sua pesquisa, deve fugir de atalhos que levarão, inevitavelmente, ao questionamento dos dados colhidos na pesquisa. Um equívoco comum, que se observa em algumas pesquisas nacionais, é usar escalas traduzidas sem considerar suas qualidades de medida em nossa cultura, ou seja, sem a realização prévia de seu estudo psicométrico. Outra confusão é criar uma "nova escala" com o ajuntamento de partes de escalas já existentes ou ainda com a modificação dos itens. Esse atalho fragiliza a coerência teórica subjacente ao instrumento e, essa escala "retalhada", poderia não avaliar com fidelidade o construto sob estudo (Campana \& Tavares, 2009; Thompson, 2004).

Criar uma nova escala ou adaptar transculturalmente e validar uma escala internacional para o Brasil são estratégias mais assertivas para o pesquisador que não encontrar um instrumento adequado para usar em sua pesquisa (Beaton, Bombardier, Guillemin, \& Ferraz, 2002). É melhor seguir por esses caminhos mais longos e custosos, assegurando-se da coerência teórica do instrumento, de sua consistência interna e validade, o que contribuirá para a qualidade dos dados.

\section{Procedimentos na coleta de dados}

Na coleta de dados, é importante determinar um protocolo e ser fiel ao seu planejamento. O protocolo de coleta de dados deverá sempre considerar os objetivos da pesquisa, organizando a coleta de maneira a que os dados sejam obtidos de forma a propiciar informações de qualidade para responder às hipóteses e questionamento levantados na pesquisa.

A ordem dos instrumentos a serem aplicados é um quesito importante, especialmente, se houver algum tipo de avaliação antropométrica envolvida, ou prática de exercícios, ou refeição ou troca de roupa ou mudança de iluminação 
no ambiente. Se algum desses fatores de "barulho" estiver envolvido, dê preferência a aplicar os questionário atitudinais ou fazer sua avaliação perceptiva antes que o participante da pesquisa seja exposto a eles (McCabe et al., 2006; Smeets, 1997). Por exemplo, numa pesquisa com pessoas que praticam exercício regulamente, o pesquisador poderá determinar que a coleta de dados ocorre pelo menos 24 horas antes e 24 horas depois de qualquer atividade física, e seguir dessa forma por todo o estudo. É isso que tornará possível controlar a variável de "barulho", que é o exercício físico. Isso é requisito para a pesquisa que esteja focada em avaliar traços da Imagem Corporal. Se seu objetivo for o de investigar os efeitos imediatos destes e de outros fatores na representação do corpo, o procedimento é inverso. Seguindo uma variação do exemplo anterior, o pesquisador que estiver interessado em saber os efeitos imediatos (estado) do exercício físico na Imagem Corporal, deverá colher dados logo ao final de uma sessão de exercícios, pois é o efeito imediato da variável de barulho que interessa (que nesse caso seria de fato uma variável dependente).

Ainda quanto à ordem dos instrumentos, outro ponto deve ser ressaltado. Caso o estudo-piloto da pesquisa/ pré-teste dos instrumentos de medida indique que a ordem dos instrumentos de avaliação (sejam eles perceptivos e/ou atitudinais) tem influência sobre a resposta dos questionários, o pesquisador deve balancear a ordem dos instrumentos, de forma a eliminar esse efeito. Por exemplo, numa pesquisa que recrutará 600 participantes, em que (1) Body Shape Questionnaire, (2) Body Esteem Scale e (3) Adaptive Probit Estimation são os instrumentos de coleta de dados e ficou evidenciado que um ou mais têm influência sobre a resposta dos demais (pelos relatos dos participantes envolvidos no pré-teste/piloto), 100 participantes responderão na ordem $1-2-3 ; 100$ na ordem $3-1-2 ; 100$ na ordem $2-3-1 ; 100$ na ordem $1-3-2 ; 100$ na ordem 3-2-1; e finalmente, os demais 100 participantes da pesquisa, na ordem 2-1-3. Esse procedimento de balanceamento deve ser reportado no relato da pesquisa.

As instruções dadas aos participantes é outro ponto importante, mas de especial destaque nas pesquisas que avaliam a dimensão perceptiva da Imagem Corporal, pois via de regra, são instruções orais (Gardner, 2011; Smeets, 1997; Tavares, Campana, Tavares-Filho \& Campana, 2010; Thompson \& Dolce, 1989). Resumidamente, apontamos alguns elementos que podem auxiliar na elaboração do protocolo de coleta de dados numa pesquisa perceptiva (Gardner, 1996; Smeets, 1997; Tavares et al., 2010; Thompson \& Gardner, 2002):

(1)Determine um horário para coleta de dados: manhã, tarde ou noite. Se os horários foram variados, procure balancear seu número amostral nos horários;

(2) $\mathrm{Na}$ medida do possível, procure controlar, ou pelo menos registrar para considerar na discussão dos dados, o intervalo entre o teste e o engajamento em possíveis variáveis intervenientes (como a última refeição e a última sessão de atividade física, por exemplo);

(3) Há alguns fatores pessoais que também interferem na avaliação perceptiva e igualmente, devem ser registrados

5 Para uma melhor compreensão do conceito de variáveis de barulho recomendamos a leitura de Simões e Tiedemann (1985). para coleta de dados. Destacamos o índice de massa corporal, o percentual de gordura corporal, sensação de dor, estado de humor, marcas na face e data da última menstruação (no caso de participantes do sexo feminino);

(4) Determine se sua avaliação perceptiva avaliará com maior ênfase os componentes mais "cognitivos" ou mais "afetivos" da percepção, ou se as duas possibilidades serão exploradas no estudo. Se esta última for a escolha, mas uma vez, recomenda-se que a coleta de dados seja balanceada quanto à ordem da coleta de informações perceptivas;

(5) Cuide e mantenha um padrão no ambiente da coleta de dados: mantenha luminosidade da sala, procure criar uma sala sem marcas ou pontos de referências de variação de tamanho (como colunas, batentes) nas paredes, sem enfeites que possam ser usados também como referências externas (como vasos), com poucos ruídos externos e estabeleça um tipo de roupa a ser usada durante o teste;

(6) Mantenha sempre a mesma pessoa na condução dos testes, e se isso não for possível, pelo menos que os avaliadores sejam do mesmo sexo. Assegure-se de que todos darão as mesmas instruções aos participantes para os testes. Esta e as demais recomendações lhe ajudarão a controlar as variáveis externas que interferem na avaliação perceptiva.

Por fim, ressalta-se que, assim como na avaliação perceptiva, conforme recomendação (5), na avaliação dos componentes da dimensão atitudinal é necessário estabelecer qual será o local de coleta de dados. Uma coleta realizada numa sala quieta, com espaço suficiente entre os respondentes, de forma a assegurar a confidencialidade das respostas, gerará dados diferentes de uma coleta realizada numa sala de espera de hospital, com pessoas ao redor. De toda forma, se a coleta de dados em locais diversos ou pouco privados for difícil de evitar, não se esqueça de esclarecer essa informação nos procedimentos e de considerar esse fato na discussão dos dados.

\section{Análise dos dados}

O primeiro passo na análise de dados quantitativos é verificar a distribuição dos dados. Como a pesquisa em Imagem Corporal lida sobretudo com atitudes humanas, é esperado que os dados tenham uma distribuição não aderente à distribuição normal, todavia é importante assegurar-se do tipo de distribuição dos dados para a escolha adequada dos testes estatísticos inferenciais. Caso o pesquisador proceda com alguma forma de transformação de dados - para garantir a normalidade ou a homocedasticidade, ou ainda a dependência linear entre as variáveis - é interessante, e mais cientificamente correto, descrever esses procedimentos.

A média e desvio padrão, ou a mediana e o range, dos escores das escalas envolvidas devem ser apresentados, de forma a propiciar a comparação dos dados do estudo com outros já desenvolvidos, ou que serão realizados no futuro. $\mathrm{O}$ pesquisador deve ficar atento às instruções contidas na forma de fazer o escore final da escala: em escalas com mais de um fator, via de regra, o pesquisador é instruído a calcular o escore de cada fator da escala. Mas há variações 
e, por isso, o estudo de desenvolvimento da escala deve ser lido atentamente para identificar a melhor forma de obter o resultado final da escala e trabalhar com os dados. Por exemplo, a Drive for Muscularity Scale, quando usada em amostras masculinas, deve ter o escore de cada fator calculado. Quando utilizada em amostras femininas, o escore é geral, ou seja, a soma de todos os itens. Em estudo de comparação de amostras femininas e masculinas, trabalha-se apenas com o escore geral.

Deve-se dar atenção aos itens reversos. A não inversão do escore do item reverso atrapalhará o cálculo do escore da escala, gerando resultados não condizentes com o que foi avaliado. Especialmente em escalas de figuras, calcule o nível de insatisfação com o corpo (dado pela diferença entre corpo ideal e corpo atual) em valores modulares. Quando, na análise dos dados, o cálculo da média da população não é feito em valores modulares $(|\mathrm{x}|)$, sofre o efeito da vontade de ganhar peso (marcado pelo sinal positivo $(+)$ ) e da vontade de perder peso (marcado pelo sinal negativo $(-))^{6}$. Na média dos dados, caso a amostra seja equilibrada entre aqueles que desejam perder e ganhar peso (amostras masculinas estão mais sujeitas a essa situação), parece não haver diferenças entre o corpo real e ideal, o que indica ausência de insatisfação corporal. Essa aparente igualdade entre corpo ideal e real, longe de ser sinônimo de satisfação, é o resultado da combinação matemática de dois grupos insatisfeitos.

Pesquisas qualitativas, por sua vez, exigem igual rigor no tratamento das informações colhidas. A transcrição fiel do material da entrevista ou grupo focal é o primeiro passo para uma análise de qualidade. Seguir rigorosamente os procedimentos de análise dos dados escolhidos - seja análise do discurso, análise do conteúdo, decomposição temática, entre outras disponíveis - é condição sine qua non para a geração de evidências que possam levar a conclusões relevantes. Conhecer o universo da população em estudo, conhecer profundamente os aspectos teóricos da Imagem Corporal e uma revisão de literatura consistente suportam, conjuntamente com as diretrizes do método escolhido de análise, que os dados não sejam discutidos de uma forma superficial.

Por fim, o pesquisador não deve perder de vista o fato de que "correlação não é causação", ou seja, uma análise correlacional não permite afirmar uma relação de causa e efeito. A ocorrência de correlação entre variáveis indica que estas crescem na mesma direção (associação positiva) ou decrescem em direções opostas (associação negativa) com significância estatística (ou seja, não ocorrem ao acaso). Relações causais, por sua vez, indicam que um fator tem influência sobre o outro, que um pode predizer o outro. A única forma de demonstrar causalidade é o controle das variáveis no tempo, quando é permitido avaliar a

6 Exemplificando: se em uma escala de figuras, o corpo que considero "ideal" está três silhuetas mais magras que meu corpo real, meu escore de insatisfação é - 3 . Se ao meu lado, o "ideal" de corpo de outra pessoa é exatamente três silhuetas acima da silhueta apontada como corpo "real", o escore de insatisfação desta pessoa é +3 . A média matemática de nossos escores de insatisfação é zero, ou seja, na média, parece não haver diferenças entre o corpo ideal e o corpo real, parece não haver insatisfação. Enquanto na verdade, o que ocorre, são formas distintas de insatisfação com o corpo. complexidade das relações entre as variáveis dependentes e independentes (Cliff, 1983). Todavia, o pesquisador que obtiver dados aderentes à distribuição normal pode indicar fatores preditores através de análise de regressão, sem esquecer entretanto, de informar o grau de explicação da dependência dos fatores. Outra forma de apontar relações de influência, numa interpretação de preditores x consequência, é através da Modelagem de Equações Estruturais. Mas, grandes amostras são inerentes à essa técnica estatística (o que limita seu uso) e ela apenas será benéfica ao pesquisador se uma forte teoria estiver subjacente ao modelo proposto (Mueller, 1997). O pesquisador não deve perder de vista, ao usar esta técnica estatística que, mesmo frente a um bom ajuste, não existe um único modelo para aquelas variáveis estudadas, havendo, além daquela investigada, uma variedade de formas válidas de explicar determinadas relações de regressão entre os construtos (Thompson, 2004.

\section{Conclusões e reflexões finais}

Neste artigo metodológico, foram apresentados elementos que se consideraram mais pertinentes para a pesquisa em Imagem Corporal no cenário brasileiro. Para garantir coerência e continuidade das investigações nessa área, é essencial que o pesquisador tenha conhecimento do assunto, dimensionado de forma proporcional à complexidade do tema. Em resumo, questões metodológicas como delimitação do referencial teórico, escolha de instrumentos confiáveis e adequados, atenção ao protocolo de coleta de dados e tratamento estatístico adequado, seguido por um discussão apoiada num referencial teórico consistente, são essenciais para a boa prática de pesquisa em Imagem Corporal.

Ressaltamos que tanto a pesquisa qualitativa e quantitativa podem oferecer informações sob o mesmo componente da Imagem Corporal, mas de forma distinta. São como veículos que seguem por rotas diferentes para atingir um mesmo ponto. A escolha de uma abordagem qualitativa ou quantitativa dependerá da pergunta da pesquisa e se o que se investiga está numa fase dedutiva ou indutiva.

Um conjunto mais amplo de tipos de dados, de técnicas de obtenção com controle mais baixo, de delineamentos transversais e de tratamento qualitativo dos dados pode ser muito mais apropriado para uma fase indutiva inicial. Por conduzir predições testáveis tende a ligar-se a um tipo restrito de dados, à obtenção direta e controlada de dados, a uma mistura de modelos de monitoramento de mudança e ao tratamento quantitativo dos dados (Breakwell, Hammond, Fife-Schaw, \& Smith, 2010, p. 40).

$\mathrm{Na}$ pesquisa quantitativa, trabalha-se então com um número restrito de variáveis, numa grande quantidade de dados, na qual a lógica é colocar em teste a hipótese nula da pesquisa, buscando por resultados significantes, com efeito razoável sobre a variável em estudo. Na pesquisa qualitativa, o número de variáveis levantadas é muito maior, num número menor de sujeitos, não permitindo afirmações categóricas, mas podendo ser a geradora de hipóteses para uma pesquisa quantitativa. De forma complementar, a pesquisa quantitativa pode indicar a necessidade de uma pesquisa qualitativa num 
ponto específico encontrado. Pode-se admitir uma relação complementar entre as duas abordagens.

Por fim, enfatiza-se que a pesquisa em Imagem Corporal pode ser feita por profissionais de todas as áreas, de forma ética, condizente com os limites de seu conhecimento específicos. Questões éticas relacionadas à responsabilidade com a realidade existencial do outro devem permear todo o processo de pesquisa em Imagem Corporal, independentemente do foco da pesquisa.

\section{Referências}

Beaton, D. E., Bombardier, C., Guillemin, F., \& Ferraz, M. B. (2002). Recommendations for the cross-cultural adaptation of healthy status measures. Illinois (USA): American Academy of Orthopedic Surgeons. Institute for Work \& Health.

Breakwell, G. M., Hammond, S., Fife-Schaw, C., \& Smith, J. A. (2010). Métodos de pesquisa em psicologia. Porto Alegre: Artmed.

Campana, A.N.N.B. (2011) Relações entre as dimensões da imagem corporal: um estudo com homens brasileiros. 2011. (unpublished dissertation thesis) Faculdade de Educação Física. Universidade Estadual de Campinas, Campinas, São Paulo, Brasil

Campana, A. N. N. B., \& Tavares, M. C. G. C. F. (2009). Avaliação da Imagem Corporal: instrumentos e diretrizes para pesquisa. São Paulo: Phorte Editora.

Cash, T. (2011). Crucial considerations in the assessment of body image. In T. Cash \& L. Smolak (Eds.), Body Image: a handbook of science, practice and prevention. (pp. 129-137). Nova Iorque: The Guilford Press.

Cliff, N. (1983). Some cautions concerning the application of Casual Modeling Methods. Multivariate Behavioural Research, 18, 115-126.

DeVellis, R. F. (2003). Scale development: theory and applications ( 2 ed.). Newbury Park CA: Sage Publications.

Fisher, S. (1990). The evolution of psychological concepts about the body In T. Cash \& T. Pruzinsky (Eds.), Body Images: development, deviance and change (pp. 3-20). Nova Iorque: The Guilford Press.

Gardner, R. M. (1996). Methodological issues in assessment of the perceptual component of body image disturbance. British Journal of Psychology, 87, 327-337
Gardner, R. M. (2011). Perceptual measures of body image for adolescents and adults. In: T. Cash \& L. Smolak (Eds.), Body Image: a handbook of science, practice and prevention. (pp. 146-153). Nova Iorque: The Guilford Press.

Krawczyk, R., Menzel, J., \& Thompson J. K. (2012). Methodological issues in the study of body image and appearance. In N. Rumsey \& D. Harcourt (Eds.), The Oxford handbook of the psychology of appearance (pp. 605-619). Oxford: Oxford University Press.

McCabe, M., Ricciardelli, L., Sitaram, G., \& Mikail, K. (2006). Accuracy of body size estimation: role of biopsychosocial variables. Body Image, 3, 163-171.

Menzel, J., Krawczyk, R., \& Thompson J. K. (2011). Atittudinal assessment of body image for adolescents and adults. In T. Cash \& L. Smolak (Eds.), Body Image: a handbook of science, practice and prevention. (pp. 154-172). Nova Iorque: The Guilford Press.

Mueller, R. O. (1997). Structural Equation Modeling: back to basics. Structural Equation Modeling, 4, 353-369

Pasquali, L. (2003). Psicometria: teoria dos testes na psicologia e na educação. Petrópolis: Vozes.

Simões, E. Q., \& Tiedemann, K. B. (1985). Psicologia da Percepção. São Paulo: EPU.

Smeets, M. (1997). The rise and fall of body size estimation research in anorexia nervosa: a review and reconceptualization. European Eating Disorders Review, 5, 75-95.

Tavares, M.C.G.C.F. (2003). Imagem corporal: Conceito e desenvolvimento. Barueri: Manole.

Tavares, M. C. G. C. F. Campana, A. N. N. B, Tavares-Filho, R. F., \& Campana, M. B. (2010). Avaliação perceptiva da imagem corporal: história, reconceituação e perspectivas para o Brasil. Psicologia em Estudo, 15, 509-518.

Thompson, J. K. (1990). Body image disturbance: assessment and treatment. Nova Iorque: Pergamon Press.

Thompson, J. K. (2004). The (mis) measurement of body image: ten strategies to improve assessment for applied and research purposes. Body Image, 1, 7-14.

Thompson, J. K., \& Dolce, J. (1989). The discrepancy between emotional vs. rational estimates of body size, actual size and ideal body ratings. Journal of Clinical Psychology, 45, 473-478

Thompson, J. K., \& Gardner, R. M. (2002). Measuring Perceptual Body Image among adolescents and adults. In T. Cash \& T. Pruzinsky (Eds.), Body Image: a handbook of theory, research \& clinical practice (pp.135-141). Nova Iorque: The Guilford Press.

Thompson, J.K, Heinberg, L., Altabe, M., \& Tantleff-Dunn, S. (1998). Exacting beauty: theory, assessment and treatment of body image disturbance. Washington: APA. 